\title{
MUSA-INT: Multicriteria customer satisfaction analysis with interacting criteria
}

\author{
Silvia Angilella, Salvatore Corrente*, Salvatore Greco*† Roman Słowiński ${ }^{\ddagger}$ \\ June 1, 2015
}

${ }^{*}$ Department of Economics and Business, University of Catania, Corso Italia 55, 95129 Catania, Italy, e-mails: angisil@unict.it, salvatore.corrente@unict.it, salgreco@unict.it

${ }^{\dagger}$ Portsmouth Business School, Operations \& Systems Management University of Portsmouth, Portsmouth PO1 3DE, United Kingdom

¥Institute of Computing Science, Poznań University of Technology, 60-965 Poznań, and Systems Research Institute, Polish Academy of Sciences, 01-447 Warsaw, Poland, e-mail:roman.slowinski@cs.put.poznan.pl 


\begin{abstract}
We are considering the problem of measuring and analyzing customer satisfaction concerning a product or a service evaluated on multiple criteria. The proposed methodology generalizes the MUSA (MUlticriteria Satisfaction Analysis) method. MUSA is a preference disaggregation method that, following the principle of ordinal regression analysis, finds an additive utility function representing both the comprehensive satisfaction level of a set of customers and a marginal satisfaction level with respect to each criterion. Differently from MUSA, the proposed approach, that we will call MUSA-INT, takes also into account positive and negative interactions among criteria, similarly to the multicriteria method UTA ${ }^{G M S_{-}}$INT. Our method accepts evaluations on criteria with different ordinal scales which do not need to be transformed into a unique cardinal scale prior to the analysis. Moreover, instead of a single utility function, MUSA-INT can also take into account a set of utility functions representing customers' satisfaction, adopting the robust ordinal regression methodology. An illustrative example shows how the proposed methodology can be applied on a customers' survey.
\end{abstract}

Keywords: Multiple criteria decision aiding; Customer satisfaction analysis; Utility function; Interacting criteria; Ordinal scales. 


\section{Introduction}

Customer satisfaction evaluation plays a key role in the enterprises' organization, contributing through discovery and representation of customers' preferences to the definition of different salient aspects of companies' strategies.

Among other advantages, customer satisfaction could increase companies' competitiveness [35], identify potential market opportunities, direct new actions to the quality improvement of a product or a service [32], and could also have a positive effect on brand equity [47].

Several approaches have been already developed to evaluate customer satisfaction (see [32] for a detailed list of the existing methods). The most used approaches are the statistical ones: the multiple regression analysis, the discriminant analysis, and the conjoint analysis $[26,28]$ that nowadays is one of the most important marketing research tools (see [33] for an overview and recent developments).

In conjoint analysis, customers are asked to evaluate combinations of different values of the attributes considered for a product or a service. On the basis of customers' answers, conjoint analysis aims at identifying the most desirable attribute values to be implemented in a product or a service.

Customer satisfaction analysis has also been approached using dominance-based rough set theory [19] which aims at inferring some simple decision rules from the consumers' data [20], differently from the conjoint analysis which represents customers' preferences with a comprehensive utility function.

Another interesting approach to customer satisfaction analysis consists in preference learning (see [12] for an updated state-of-the-art) that, given some preferences on a set of objects, searches a function to predict the preferences on a new set of objects. For example, some preference learning applications are provided by a search engine's ranking of web pages according to customers' preferences, or by stores' rankings of particular products according to the preferences expressed on-line by the clients.

Customers' satisfaction evaluation has also been studied from a multiple criteria point of view, using the method MUSA (MUlticriteria Satisfaction Analysis [30]). MUSA is a preference disaggregation method that, following the principle of ordinal regression analysis [37], finds an additive utility function representing the satisfaction level of a set of customers based on their expressed preferences collected in a satisfaction survey's data. Using MUSA, the customers are asked to give a comprehensive satisfaction level for a service or a product under consideration, but also a marginal satisfaction level for each one of its features (evaluation criteria). MUSA has many advantages over the traditional customer satisfaction models, since it fully considers the qualitative form of customers' judgments and preferences that are usually expressed in this way in the consumers' questionnaires. The success of MUSA is witnessed by many applications in different fields as, for example, bank sector [29], agricultural marketing [46] and transportation-communication sector [31]. Despite these positive aspects, MUSA is not able to represent positive and negative synergies between specific features of a product or a service, since it considers an additive utility function and, consequently, its underlying hypothesis is preference independence [39, 51].

This is an important issue because it is a common experience that in the evaluation of a product or a service, some features could positively or negatively interact. For example, in the evaluation of a supermarket, prices and special offers have, usually, a negative interaction. In fact, prices and special offers are both important in evaluating a supermarket, however, a supermarket with low prices has also often many special offers and thus, considering together prices and special offers, the total importance is smaller than the sum of their marginal importances. Analogously, one can say that there is a positive synergy between goods' quality and prices, because in general a supermarket with high quality of goods has also high prices and thus a supermarket with a high quality of goods and relatively low prices is well appreciated, such that the total importance of goods' quality and prices considered together is higher than the sum of the importance of their marginal importances.

In Multiple Criteria Decision Aiding (MCDA, see [9] for an updated state-of-the-art) positive and 
negative interaction among criteria are very often represented using some fuzzy integrals, such as the Choquet integral [5] or some of its generalizations, e.g., the bipolar Choquet integral ([14, 15]; see also [21]) or the level dependent Choquet integral [18], (see [16] for a survey about the use of Choquet integral in MCDA). Fuzzy integrals, and among them the Choquet integral, are aggregation models that, besides other technical assumptions, require a scale of measurement which is cardinal (more precisely, an interval scale [45]) and common to all the criteria (features) taken into consideration. Such a scale permits comparison of evaluations on different criteria, so that, e.g., it becomes possible to say that, for a given supermarket, the level of prices is better than the special offers it proposes and, moreover, these are better than the quality of the goods.

Even if the majority of conjoint analysis methods does not consider interaction among attributes [4], there are several contributions, like [1, 13, 27, 34, 40, 42, 44], that estimate by means of a statistical regression not only a value for each level of each attribute, but also a value for each combination of levels on a set of couples of attributes (possibly all couples of attributes). Another approach proposed to represent interaction among attributes in conjoint analysis is based on the use of the Choquet integral [48, 36, 41, 49, 50, 52].

Since we want to take into account not more than ordinal qualitative aspects of the scales of criteria, we propose MUSA-INT, being a generalization of the multicriteria method MUSA. MUSAINT handles positive and negative synergies between couples of criteria, using a formulation of the utility function recently proposed in the multicriteria method UTA ${ }^{G M S}$-INT [24]. Differently from the 2-additive Choquet integral aggregation model, UTA ${ }^{G M S}$-INT represents positive and negative synergies avoiding any arbitrary transformation of the original ordinal scales into a unique artificial cardinal scale.

The paper is organized as follows. In Section 2, we introduce the basic concepts and the relative notation, a brief description of the MUSA method, and the specific utility function adopted in our customer satisfaction model. In Section 3, basic steps of the proposed multicriteria customer satisfaction analysis are described. Some further extensions of the proposed method are presented in Section 4. Section 5 contains an illustrative example, considering a set of customers' satisfaction questionnaires on which MUSA-INT is applied. Conclusions and future directions of research are collected in Section 6.

\section{Basic concepts and the MUSA method}

The basic elements of the proposed methodology are the following:

- $C=\{1, \ldots, r\}$ is the set of customers,

- $I=\{1, \ldots, n\}$ is the set of evaluation criteria (features),

- $\mathcal{L}^{i}=\left\{\ell_{1}^{i}, \ldots, \ell_{s_{i}}^{i}\right\}, i=1, \ldots, n$, is the set of levels of satisfaction for criterion $i$ : for example, for a given criterion $i$, the scale could be $\mathcal{L}^{i}=\left\{\ell_{1}^{i}, \ell_{2}^{i}, \ell_{3}^{i}\right\}$, with $\ell_{1}^{i}=$ "dissatisfied", $\ell_{2}^{i}=$ "satisfied", $\ell_{3}^{i}=$ "very satisfied"; the levels $\ell_{1}^{i}, \ldots, \ell_{s_{i}}^{i}$ are increasingly ordered with respect to the satisfaction level, i.e. the satisfaction represented by $\ell_{p}^{i}$ is greater than the satisfaction represented by $\ell_{p-1}^{i}, p=2, \ldots, s_{i}$,

- $\mathcal{L}^{n+1}=\left\{\ell_{1}^{n+1}, \ldots, \ell_{s_{n+1}}^{n+1}\right\}$ is the set of levels of comprehensive satisfaction: the levels $\ell_{1}^{n+1}, \ldots, \ell_{s_{n+1}}^{n+1}$ are increasingly ordered with respect to the satisfaction level,

- $\operatorname{sat}_{c, i} \in \mathcal{L}^{i}$ is the satisfaction expressed by customer $c \in C$ with respect to criterion $i \in I$,

- $\operatorname{sat}_{c, n+1} \in \mathcal{L}^{n+1}$ is the comprehensive satisfaction expressed by the customer $c \in C$, 
- $u_{i}: \mathcal{L}^{i} \rightarrow[0,1]$ is the marginal utility function of criterion $i$,

- $U: \mathcal{L}^{n+1} \rightarrow[0,1]$ is the utility of comprehensive levels of satisfaction,

- $S y n^{+} \subseteq I^{(2)}$ with $I^{(2)}=\left\{\left\{i_{1}, i_{2}\right\} \subseteq I\right\}$ is the set of all couples of criteria for which there is a positive interaction,

- $S y n^{-} \subseteq I^{(2)}$ is the set of all couples of criteria for which there is a negative interaction,

- $\operatorname{syn}_{i j}^{+}: \mathcal{L}^{i} \times \mathcal{L}^{j} \rightarrow[0, \rho]$ is a function non-decreasing in both its two arguments representing the strength of the positive interaction between criteria $i, j \in I$, such that $\{i, j\} \in \operatorname{Syn}^{+}$( $\rho$ is a positive real constant),

- $\operatorname{syn}_{i j}^{-}: \mathcal{L}^{i} \times \mathcal{L}^{j} \rightarrow[0, \rho]$ is a function non-decreasing in both its two arguments representing the strength of the negative interaction between criteria $i, j \in I$, such that $\{i, j\} \in S_{y n^{-}}$(as above, $\rho$ is a positive real constant).

In the MUSA method [30], inspired by the idea of ordinal regression used in the UTA methods [37], one represents customer satisfaction through the following additive utility function,

$$
U\left(\operatorname{sat}_{c, n+1}\right)=\sum_{i=1}^{n} u_{i}\left(s_{c, i}\right), c \in C .
$$

The utility function (1) is obtained by solving the following LP problem [30]:

$$
\begin{gathered}
\text { Minimize: } \sum_{c=1}^{r}\left(\sigma_{c}^{+}+\sigma_{c}^{-}\right), \text {s.t. } \\
\left\{\begin{array}{l}
U\left(s_{c, n+1}\right)=\sum_{i=1}^{n} u_{i}\left(s_{c} t_{c, i}\right)-\sigma_{c}^{+}+\sigma_{c}^{-}, \text {for all } c \in C \\
\sigma_{c}^{+} \geq 0, \sigma_{c}^{-} \geq 0, \text { for all } c \in C \\
u_{i}\left(\ell_{p}^{i}\right) \geq u_{i}\left(\ell_{p-1}^{i}\right), p=2, \ldots, s_{i}, \text { for all } i \in I, \\
U\left(\ell_{p}^{n+1}\right) \geq U\left(\ell_{p-1}^{n+1}\right), p=2, \ldots, s_{n+1}, \\
u_{i}\left(\ell_{1}^{i}\right)=0, \text { for all } i \in I, \\
\sum_{i=1}^{n} u_{i}\left(\ell_{s_{i}}^{i}\right)=1, \\
U\left(\ell_{s_{n+1}}^{n+1}\right)=1,
\end{array}\right\} \text { (monotonicity conditions) }
\end{gathered}
$$

where $\sigma_{c}^{+}$and $\sigma_{c}^{-}$are over- and under-estimation errors for every customer's utility function.

Differently from MUSA, the utility function considered in our model is the one proposed in the multicriteria method UTA ${ }^{G M S}$-INT (see [24]), which takes into account positive and negative interactions between couples of criteria as follows:

$$
U\left(\operatorname{sat}_{c, n+1}\right)=\sum_{i=1}^{n} u_{i}\left(s a t_{c, i}\right)+\sum_{\{i, j\} \in S y n^{+}} \operatorname{syn}_{i j}^{+}\left(s a t_{c, i}, s a t_{c, j}\right)-\sum_{\{i, j\} \in S y n^{-}} \operatorname{syn}_{i j}^{-}\left(s a t_{c, i}, s a t_{c, j}\right), c \in C .
$$




\section{Description of MUSA-INT}

In this section, we present a new procedure for finding a utility function representing the comprehensive satisfaction of a set of customers $C$. The adopted utility function defined by (3) handles synergies between satisfaction levels on two criteria, $i$ and $j: s a t_{c, i}$ and $s a t_{c, j}$.

The multicriteria customer satisfaction analysis we are proposing is composed of three main successive phases:

(i) finding a utility function $U$ representing the satisfaction of all customers from set $C$ with a minimal sum of approximation errors;

(ii) identifying a minimal set of couples of interacting criteria, where minimality is referred to the inclusion;

(iii) finding a utility function discriminating as much as possible satisfaction levels for both marginal and comprehensive utility functions.

From a computational point of view, each phase consists in solving a specific mixed integer linear program (MILP). Let us examine each phase in detail.

\subsection{Phase (i): finding a utility function representing the satisfaction of all the customers}

Since we want to get a utility function $U$ representing the utility of all customers from set $C$ with a minimal sum of approximation errors, we need to introduce non-negative error variables $\sigma_{c}^{+}, \sigma_{c}^{-} \geq 0$, corresponding to over- and under-estimation, respectively, for every customer's utility as follows:

$$
U\left(s a t_{c, n+1}\right)=\sum_{i=1}^{n} u_{i}\left(s a t_{c, i}\right)+\sum_{\{i, j\} \in I^{(2)}} \operatorname{syn}_{i j}^{+}\left(s a t_{c, i}, s a t_{c, j}\right)-\sum_{\{i, j\} \in I^{(2)}} \operatorname{syn}_{i j}^{-}\left(s a t_{c, i}, s a t_{c, j}\right)-\sigma_{c}^{+}+\sigma_{c}^{-},
$$

for all $c \in C$.

The objective function to be minimized is the sum of the error variables over all customers from set $C$ (analogically to the original UTASTAR method [37]):

$$
\sum_{c=1}^{r}\left(\sigma_{c}^{+}+\sigma_{c}^{-}\right)
$$

In the same spirit of what was done in $\mathrm{UTA}^{G M S}$-INT [24], we consider the following options for the positive and negative interactions present in the value function (4) for each couple of criteria $\{i, j\} \in I^{(2)}$ :

(S1) $\operatorname{syn}_{i j}^{+}\left(s a t_{c, i}, s a t_{c, j}\right)$ and $\operatorname{syn}_{i j}^{-}\left(s a t_{c, i}, s a t_{c, j}\right)$ are not mutually exclusive, such that in the evaluation space of the two criteria there is a switch between positive interaction and negative interaction; in some parts of the space the positive interaction prevails and in some others a negative interaction prevails, or even, there is no interaction,

(S2) $\operatorname{syn}_{i j}^{+}\left(s a t_{c, i}, s a t_{c, j}\right)$ and $\operatorname{syn}_{i j}^{-}\left(s a t_{c, i}, s a t_{c, j}\right)$ are mutually exclusive,

(S3) only one of the two interactions is considered, for example the positive one. 
According to [24], in order to ensure the monotonicity of the utility function, we consider for all $J \subseteq I$ the following constraint: if $s a t_{c, i} \geq s a t_{d, i}$, for all $i \in J$, and $c, d \in C$, then

$$
\begin{aligned}
& \sum_{i \in J} u_{i}\left(s a t_{c, i}\right)+\sum_{\{i, j\} \subseteq J} \operatorname{syn}_{i j}^{+}\left(\operatorname{sat}_{c, i}, s a t_{c, j}\right)-\sum_{\{i, j\} \subseteq J} s y n_{i j}^{-}\left(s_{c} t_{c, i}, s a t_{c, j}\right) \geq \\
& \geq \sum_{i \in J} u_{i}\left(s a t_{d, i}\right)+\sum_{\{i, j\} \subseteq J} s y n_{i j}^{+}\left(s a t_{d, i}, s a t_{d, j}\right)-\sum_{\{i, j\} \subseteq J} s y n_{i j}^{-}\left(s a t_{d, i}, s a t_{d, j}\right) .
\end{aligned}
$$

Someone could object that constraint (6) could be obtained more easily by imposing the same constraint only for all couples of criteria $\{i, j\} \in I^{(2)}$. This is not true, because it is possible that constraint (6) is verified for each couple of criteria $\{i, j\} \in I^{(2)}$, but the same constraint is not true for a subset of criteria $J \subseteq I,|J|>2$, for which $s a t_{c, i} \geq s a t_{d, i}$ for all $i \in J$, and $c, d \in C$.

Because constraints described by inequality (6) are numerous, and we would like to consider as simple model as possible, i.e. with the lowest possible number of interactions, we shall suppose, that each criterion $i$ can interact with at most one another criterion. Under this assumption, constraint (6) has to be considered only for each $J \subseteq I$, where $|J|=2$.

In case of option (S1), for each couple of criteria $\{i, j\} \in I^{(2)}$, the following binary variable $\gamma_{i j}$ is introduced:

$$
\gamma_{i j}= \begin{cases}1 & \text { if }\{i, j\} \in I^{(2)} \text { are interacting, } \\ 0 & \text { if }\{i, j\} \in I^{(2)} \text { are not interacting. }\end{cases}
$$

Thus, the following constraints are considered in the first MILP problem:

$$
E_{(S 1)}\left\{\begin{array}{l}
\gamma_{i j} \in\{0,1\} \\
\sum_{j \in I \backslash\{i\}} \gamma_{i j} \leq 1, \text { for all } i \in I, \\
\operatorname{syn}_{i j}^{+}\left(\ell_{s_{i}}^{i}, \ell_{s_{j}}^{j}\right) \leq \rho \gamma_{i j}, \\
\operatorname{syn}_{i j}^{-}\left(\ell_{s_{i}}^{i}, \ell_{s_{j}}^{j}\right) \leq \rho \gamma_{i j},
\end{array}\right.
$$

where, as said before, $\rho$ is an upper bound for $s y n_{i j}^{+}$and $s y n_{i j}^{-}$, e.g. equal to 1 , and the second constraint ensures that each criterion can interact with at most one another criterion.

In case of option (S2), we introduce as many binary variables $\delta_{i j}^{+}, \delta_{i j}^{-} \in\{0,1\}$ as twice the couples of criteria, i.e. $2 \times\left(\begin{array}{l}n \\ 2\end{array}\right)$. The meaning of every binary variable is the following:

$$
\delta_{i j}^{+}\left(\delta_{i j}^{-}\right)= \begin{cases}1 & \text { if }\{i, j\} \in I^{(2)} \text { are positively (negatively) interacting, } \\ 0 & \text { if }\{i, j\} \in I^{(2)} \text { are not positively (negatively) interacting. }\end{cases}
$$

For every couple of criteria $\{i, j\} \in I^{(2)}$, three situations can arise:

1) $i$ and $j$ are interacting positively $\left(\delta_{i j}^{+}=1\right)$,

2) $i$ and $j$ are interacting negatively $\left(\delta_{i j}^{-}=1\right)$,

3) $i$ and $j$ are not interacting $\left(\delta_{i j}^{+}=\delta_{i j}^{-}=0\right)$.

In consequence, the following constraints are included in the first MILP problem: 


$$
E_{(S 2)}\left\{\begin{array}{l}
\delta_{i j}^{+}, \delta_{i j}^{-} \in\{0,1\} \\
\delta_{i j}^{+}+\delta_{i j}^{-} \\
\operatorname{syn}_{i j}^{+}\left(\ell_{s_{i}}^{i}, \ell_{s_{j}}^{j}\right) \leq 1, \text { for all }\{i, j\} \in I^{(2)}, \\
\operatorname{syn}_{i j}^{-}\left(\ell_{s_{i}}^{i}, \ell_{s_{j}}^{j}\right) \leq \rho \delta_{i j}^{+}, \\
\sum_{j \in I \backslash\{i\}}\left(\delta_{i j}^{+}+\delta_{i j}^{-}\right) \leq 1 \text { for all } i \in I,
\end{array}\right.
$$

where $\rho$ can be set, e.g. equal to 1 ; the second constraint avoids that there could be positive and negative interactions for the same couple of criteria and the last constraint ensures that each criterion can interact with at most another criterion only.

In order to simplify the notation, we shall describe the set of constraints of option (S3) starting from set of constraints of the option (S2):

- if we consider only positive interactions, then the corresponding set of constraints $E_{\left(S 3^{+}\right)}$is obtained from $E_{(S 2)}$ by adding $\delta_{i j}^{-}=0$ for all $\{i, j\} \in I^{(2)}$,

- if we consider only negative interactions, then the corresponding set of constraints $E_{\left(S 3^{-}\right)}$is obtained from $E_{(S 2)}$ by adding $\delta_{i j}^{+}=0$ for all $\{i, j\} \in I^{(2)}$.

Finally, the MILP formulation includes some technical constraints concerning monotonicity and boundary conditions on the synergies, marginal utilities and comprehensive utility. In particular, monotonicity constraints ensure that the marginal utility $u_{i}\left(l_{p}^{i}\right)$ for all $i=1, \ldots, n$, and $p=1, \ldots, s_{i}$, and the comprehensive utility $U\left(l_{p}^{n+1}\right), p=1, \ldots, s_{n+1}$, are non-decreasing functions of $l_{p}^{i}$ and $l_{p}^{n+1}$, respectively, while interaction functions $\operatorname{syn}_{i j}^{+}\left(l_{p_{1}}^{i}, l_{q_{1}}^{j}\right), \operatorname{syn}_{i j}^{-}\left(l_{p_{1}}^{i}, l_{q_{1}}^{j}\right)$ are non-decreasing functions of both their two arguments $l_{p_{1}}^{i}, l_{q_{1}}^{j}$, for all $\{i, j\} \in I^{(2)}, p_{1}=1, \ldots, s_{i}$, and $q_{1}=1, \ldots, s_{j}$. Boundary conditions ensure, instead, that $U(c) \in[0,1]$ for each $c \in C$, imposing that the utility of the profile presenting the worst satisfaction on each criterion is equal to zero, while the utility of the profile presenting the best satisfaction on each criterion is equal to one.

In consequence, the set of constraints common to all the options described before is the following: 
$U\left(s a t_{c, n+1}\right)=\sum_{i=1}^{n} u_{i}\left(s a t_{c, i}\right)+\sum_{\{i, j\} \in I^{(2)}} \operatorname{syn}_{i j}^{+}\left(s a t_{c, i}, s a t_{c, j}\right)-\sum_{\{i, j\} \in I^{(2)}} \operatorname{syn}_{i j}^{-}\left(s a t_{c, i}, s a t_{c, j}\right)-\sigma_{c}^{+}+\sigma_{c}^{-}$

for all $c \in C$,

$u_{i}\left(\ell_{p}^{i}\right) \geq u_{i}\left(\ell_{p-1}^{i}\right), p=2, \ldots, s_{i}$, for all $i \in I$,

$U\left(\ell_{p}^{n+1}\right) \geq U\left(\ell_{p-1}^{n+1}\right)+\varepsilon, p=2, \ldots, s_{n+1}$,

$\operatorname{syn}_{i j}^{+}\left(\ell_{p_{1}}^{i}, \ell_{q_{1}}^{j}\right) \geq \operatorname{syn}_{i j}^{+}\left(\ell_{p_{2}}^{i}, \ell_{q_{2}}^{j}\right)$,

$\operatorname{syn}_{i j}^{-}\left(\ell_{p_{1}}^{i}, \ell_{q_{1}}^{j}\right) \geq \operatorname{syn}_{i j}^{-}\left(\ell_{p_{2}}^{i}, \ell_{q_{2}}^{j}\right)$,

$u_{i}\left(\ell_{p_{1}}^{i}\right)+u_{j}\left(\ell_{q_{1}}^{j}\right)+\operatorname{syn}_{i j}^{+}\left(\ell_{p_{1}}^{i}, \ell_{q_{1}}^{j}\right)-\operatorname{syn}_{i j}^{-}\left(\ell_{p_{1}}^{i}, \ell_{q_{1}}^{j}\right) \geq$

(monotonicity conditions),

$\geq u_{i}\left(\ell_{p_{2}}^{i}\right)+u_{j}\left(\ell_{q_{2}}^{j}\right)+\operatorname{syn}_{i j}^{+}\left(\ell_{p_{2}}^{i}, \ell_{q_{2}}^{j}\right)-\operatorname{syn}_{i j}^{-}\left(\ell_{p_{2}}^{i}, \ell_{q_{2}}^{j}\right)$,

with $p_{1} \geq p_{2}$ and $q_{1} \geq q_{2}$,

$p_{1}, p_{2}=1, \ldots, s_{i}, q_{1}, q_{2}=1, \ldots, s_{j}$, for all $\{i, j\} \in I^{(2)}$,

$u_{i}\left(\ell_{1}^{i}\right)=0$, for all $i \in I, U\left(\ell_{1}^{n+1}\right)=0$,

$\operatorname{syn}_{i j}^{-}\left(\ell_{1}^{i}, \ell_{1}^{j}\right)=0, \operatorname{syn}_{i j}^{+}\left(\ell_{1}^{i}, \ell_{1}^{j}\right)=0$, for all $\{i, j\} \in I^{(2)}$,

$\sum_{i=1}^{n} u_{i}\left(\ell_{s_{i}}^{i}\right)+\sum_{\{i, j\} \in I^{(2)}} \operatorname{syn}_{i j}^{+}\left(\ell_{s_{i}}^{i}, \ell_{s_{j}}^{j}\right)-\sum_{\{i, j\} \in I^{(2)}} \operatorname{syn}_{i j}^{-}\left(\ell_{s_{i}}^{i}, \ell_{s_{j}}^{j}\right)=1$

(boundary condition),

where $\varepsilon$ is an arbitrarily small positive quantity.

Depending on the choice of the option (S1), or (S2) or (S3), we set $\left(E_{1}\right)=E_{*} \cup E_{(\cdot)}$, where $E_{(\cdot)}$ is one of the sets of constraints: $E_{(S 1)}, E_{(S 2)}, E_{\left(S 3^{+}\right)}$, and $E_{\left(S 3^{-}\right)}$, previously defined. Some computational details on the set of constraints $\left(E_{1}\right)$ are presented in the Appendix.

It is important to pay attention to the selected value of $\varepsilon$ since it may affect the feasibility of $\left(E_{1}\right)$ or the provided solution. For this reason, it is recommended to use different values of $\varepsilon$ and select the one giving the minimum approximation error. The analyst should assist the selection of $\varepsilon$.

In order to check the existence of a utility function representing the satisfaction of all customers from set $C$, we have to solve the following MILP problem:

$$
\text { Minimize: } \sum_{c=1}^{r}\left(\sigma_{c}^{+}+\sigma_{c}^{-}\right) \text {, s.t. }
$$

$$
\left(E_{1}\right) \text {. }
$$

In real-word applications it is rather difficult to minimize the objective function to zero. For this reason, the analyst should fix the maximum acceptable error, called maxerror.

The above mixed-integer linear program returns the utility function $U$ and, moreover, for option (S1) the set Syn of couples of criteria that can interact positively and negatively, and for options (S2) and (S3), the sets $\mathrm{Syn}^{+}$and $\mathrm{Syn}^{-}$of couples of positively and negatively interacting criteria, defined, respectively, as follows:

$$
\begin{aligned}
& \text { Syn }=\left\{\{i, j\} \in I^{(2)}: \gamma_{i j}=1\right\}, \\
& \text { Syn }^{+}=\left\{\{i, j\} \in I^{(2)}: \delta_{i j}^{+}=1\right\}, S y n^{-}=\left\{\{i, j\} \in I^{(2)}: \delta_{i j}^{-}=1\right\} .
\end{aligned}
$$


Let us remark that if program (9) gives $\gamma_{i j}=0$ for option (S1), or $\delta_{i j}^{+}=\delta_{i j}^{-}=0$, for all $\{i, j\} \in I^{(2)}$ for options (S2) and (S3), i.e. when there are no interactions, the obtained utility function is the same as the one supplied by the MUSA method [30]. For this reason, MUSA-INT is a true generalization of MUSA.

If the objective function of program (9) can be minimized to zero, then there exists at least one utility function $U$, having the form of (3), representing the satisfaction of customers expressed by set of constraints $\left(E_{1}\right)$; otherwise, if the minimum value of objective function of $(9)$ is positive, then there is no utility function $U$, having the form of (3), able to represent satisfaction of customers expressed by set of constraints $\left(E_{1}\right)$.

In the latter case, let opterr be the optimal value of the objective function $\sum_{c=1}^{r}\left(\sigma_{c}^{+}+\sigma_{c}^{-}\right)$resulting from (9). Two situations can arise:

- If opterr $\leq$ maxerror, that is the error obtained in the previous phase is acceptable for the analyst, then one can pass to phase (ii) described in Section 3.2.

- If opterr > maxerror, then one can increase the maximum number of criteria with which each criterion can interact, until problem (9) gives a solution with opterr $\leq$ maxerror. Let us suppose that each criterion can interact with at most a fixed number of criteria denoted by $\eta$. In this case, we need to add the following constraints to $\left(E_{*}\right)$ :

$$
\begin{aligned}
& \sum_{i \in J} u_{i}\left(l_{p_{i}}^{i}\right)+\sum_{\{i, j\} \subseteq J} \operatorname{syn}_{i j}^{+}\left(l_{p_{i}}^{i}, l_{p_{j}}^{j}\right)-\sum_{\{i, j\} \subseteq J} \operatorname{syn}_{i j}^{-}\left(l_{p_{i}}^{i}, l_{p_{j}}^{j}\right) \geq \\
& \geq \sum_{i \in J} u_{i}\left(l_{q_{i}}^{i}\right)+\sum_{\{i, j\} \subseteq J} \operatorname{syn}_{i j}^{+}\left(l_{q_{i}}^{i}, l_{q_{j}}^{j}\right)-\sum_{\{i, j\} \subseteq J} \operatorname{syn}_{i j}^{-}\left(l_{q_{i}}^{i}, l_{q_{j}}^{j}\right),
\end{aligned}
$$

if $p_{i} \geq q_{i}$, for all $i \in J:|J|=\eta+1$ and $p_{i}=1, \ldots, s_{i}, q_{i}=1, \cdots, s_{i}$.

Moreover, constraints

$\sum_{j \in I \backslash\{i\}} \gamma_{i j} \leq 1$ for all $i \in I$ in $\left(E_{(S 1)}\right)$,

and

$\sum_{j \in I \backslash\{i\}} \delta_{i j}^{+}+\delta_{i j}^{-} \leq 1$ for all $i \in I$ in $\left(E_{(S 2)}\right)$,

should be, respectively, replaced by

$$
\sum_{j \in I \backslash\{i\}} \gamma_{i j} \leq \eta
$$

and

$\sum_{j \in I \backslash\{i\}} \delta_{i j}^{+}+\delta_{i j}^{-} \leq \eta$

\subsection{Phase (ii): identifying of a minimal set of couples of interacting criteria}

The set Syn of couples of criteria that can interact positively and negatively, or the pair $\left(S y n^{+}, S y n^{-}\right)$ of sets of couples of interacting criteria, obtained in phase (i), is not necessarily minimal, in the sense that there could exist other sets $S y n^{\prime}$ or $S y n^{\prime+}$ and $S y n^{\prime-}$ of couples of positively or negatively 
interacting criteria that could represent the utility of all customers with the same or similar approximation error $\sum_{c=1}^{r}\left(\sigma_{c}^{+}+\sigma_{c}^{-}\right)$, and such that $\operatorname{Syn} n^{\prime} \subseteq$ Syn for option (S1) or $\operatorname{Syn}^{\prime} \subseteq^{+} \operatorname{Syn}^{+}$and Syn $^{\prime-} \subseteq S y n^{-}$for options (S2) and (S3), with at least one of the two inclusions being strict.

In order to identify a minimal set $S y n$ or a minimal pair $\left(S y n^{+}, S y n^{-}\right)$of sets of couples of interacting criteria, while possibly accepting a small deterioration of the approximation error resulting from the previous phase, we have to solve the following MILP problem

Minimize: $f$, s.t.

$$
\left.\begin{array}{c}
\left(E_{1}\right) \\
\sum_{c=1}^{r}\left(\sigma_{c}^{+}+\sigma_{c}^{-}\right) \leq \text {opterr }+\alpha
\end{array}\right\}\left(E_{2}\right)
$$

where $f=\sum_{\{i, j\} \in I^{(2)}} \gamma_{i j}$ for option (S1) or $f=\sum_{\{i, j\} \in I^{(2)}}\left(\delta_{i j}^{+}+\delta_{i j}^{-}\right)$for options (S2), (S3), opterr is the optimal value of the total approximation error $\sum_{c=1}^{r}\left(\sigma_{c}^{+}+\sigma_{c}^{-}\right)$resulting from the solution of $(9)$, and $0 \leq \alpha \leq$ maxerror - opterr is a tolerance parameter controlling the possible deterioration of the total optimal approximation error.

The parameter $\alpha$ controls the trade-off between the number of criteria interacting and the total approximation error of the utility function. The analyst can tune $\alpha$ depending on his/her acceptance of the number of interacting criteria. In fact, $\mathrm{s}(\mathrm{he})$ could be interested in working with a small number of interacting criteria, giving up to a utility function more adequate to the customers' survey. Such analyst's attitude can be justified by the fact that it is easier to improve the customer satisfaction by focusing on a smaller number of interacting criteria.

In result of solving MILP problem (11), one gets a utility function $U$ (possibly different from the utility function resulting from (9)) and depending, on the option (S1), (S2), or (S3), Syn or a minimal pair $\left(\mathrm{Syn}^{+}, \mathrm{Syn}^{-}\right)$of sets of couples of positively and negatively interacting criteria, minimal in the sense of inclusion.

\subsection{Phase (iii): finding the most discriminating utility function}

In order to find a utility function $U$ (possibly different from the one obtained in the previous phase) discriminating as much as possible all levels of satisfaction by the marginal utility functions $u_{i}(\cdot)$, or by the comprehensive utility function $U(\cdot)$, while keeping the same number of interacting couples of criteria, as obtained from (11), one has to solve two MILP problems. The first one tends to discriminate as much as possible the satisfaction levels of the comprehensive utility function:

$$
\text { Maximize: } \varepsilon=\varepsilon_{\text {comprehensive, }} \text { s.t. }
$$

$$
\left.\begin{array}{c}
\sum_{\substack{\{i, j\} \in I^{(2)} \\
\gamma_{i j}}} \leq \text { optsyn, for (S1), } \\
\sum_{\{i, j\} \in I^{(2)}}\left(\delta_{i j}^{+}+\delta_{i j}^{-}\right) \leq \text {optsyn, for (S2) and (S3) }
\end{array}\right\}
$$


where $\varepsilon$ is a variable present in the constraint $U\left(\ell_{p+1}^{n+1}\right) \geq U\left(\ell_{p}^{n+1}\right)+\varepsilon$, opterr and $\alpha$ have the same meaning as in program (11), while optsyn is the optimal value of the objective function of program (11).

The solution of MILP problem (12) gives a utility function maximizing the minimal difference $U\left(\ell_{p}^{n+1}\right)-U\left(\ell_{p-1}^{n+1}\right), p=2, \ldots, s_{n+1}$. In fact, the minimum of those differences is equal to $\varepsilon_{\text {comprehensive }}$, i.e the optimal value of $\varepsilon$ given by program (12).

The analyst could be interested in finding the most discriminating function not only with respect to the comprehensive utility, but also with respect to the marginal utilities. In order to find such discriminating marginal utility functions, one has to solve the following MILP problem:

$$
\text { Maximize: } \varepsilon=\varepsilon_{\text {marginal }} \text {, s.t. }
$$

$$
\begin{aligned}
& \left.\sum_{\{i, j\} \in I^{(2)}} \gamma_{i j} \leq \begin{array}{c}
\left(E_{2}^{\prime}\right), \\
\text { optsyn, for (S1), }
\end{array}\right\}\left(E_{3}^{\prime}\right) \\
& \sum_{\{i, j\} \in I^{(2)}}\left(\delta_{i j}^{+}+\delta_{i j}^{-}\right) \leq \text {optsyn, for (S2) and (S3) }
\end{aligned}
$$

where $\left(E_{2}^{\prime}\right)$ is composed of the same constraints as $\left(E_{2}\right)$, apart from constraints

- $u_{i}\left(\ell_{p}^{i}\right) \geq u_{i}\left(\ell_{p-1}^{i}\right), p=2, \ldots, s_{i}$, for all $i \in I$,

- $U\left(\ell_{p}^{n+1}\right) \geq U\left(\ell_{p-1}^{n+1}\right)+\varepsilon, p=2, \ldots, s_{n+1}$,

that are replaced by

- $u_{i}\left(\ell_{p}^{i}\right) \geq u_{i}\left(\ell_{p-1}^{i}\right)+\varepsilon, p=2, \ldots, s_{i}$, for all $i \in I$,

- $U\left(\ell_{p}^{n+1}\right) \geq U\left(\ell_{p-1}^{n+1}\right)+\varepsilon_{\text {comprehensive }} \times(1-\beta), p=2, \ldots, s_{n+1}$,

with $\beta \in[0,1]$ representing the percentage of the comprehensive discrimination threshold that the analyst is ready to lose in order to gain on discrimination with respect to the marginal utilities.

Even in this case, the parameter $\beta$ can be tuned with the aim of increasing the discrimination with respect to the marginal utilities, since the analyst could wish to focus on the marginal utilities, instead of the comprehensive utility. Consequently, the analyst could direct his/her actions to the marginal features (criteria) to improve the customer satisfaction.

Let us observe that the sequence of the resolution of the two problems (12) and (13) is not the only possible. One could also decide to maximize first the marginal utility and then the global utility, or maximize both types of utilities at the same time.

In some cases, in order to find a discriminating utility function, both with respect to the comprehensive and the marginal satisfaction levels, it may be necessary to increase the admissible total approximation error or increase the number of interacting couples of criteria. In this case, one needs to solve again the two optimization problems (12) and (13), increasing the chosen value of $\alpha$ in $\left(E_{2}\right)$ or substituting the constraints $\sum_{\{i, j\} \in I^{(2)}} \gamma_{i j} \leq$ optsyn for option $(\mathrm{S} 1)$ or $\sum_{\{i, j\} \in I^{(2)}}\left(\delta_{i j}^{+}+\delta_{i j}^{-}\right) \leq$optsyn for options (S2) and (S3), with $\sum_{\{i, j\} \in I^{(2)}} \gamma_{i j} \leq($ optsyn $+\gamma)$ and $\sum_{\{i, j\} \in I^{(2)}}\left(\delta_{i j}^{+}+\delta_{i j}^{-}\right) \leq($optsyn $+\gamma)$, respectively, where $\gamma$ represents the number of additional interactions accepted by the analyst.

In Section 4, we shall describe how to identify alternative minimal sets $\operatorname{Syn}$ or pairs $\left(\operatorname{Syn}^{+}, S_{y n}^{-}\right)$ of sets of couples of interacting criteria being compatible with a fixed tolerance parameter $\alpha$. In case 
of multiple minimal sets $S y n$ for option (S1) or pairs $\left(S y n^{+}, S y n^{-}\right)$for options (S2) and (S3), it is interesting to compute the intersection of all the sets $S y n, S y n^{+}$and of all the sets $S_{y n}^{-}$, without deteriorating the approximation error. Let us observe that this interpretation of alternative minimal sets Syn or minimal pairs $\left(S y n^{+}, S y n^{-}\right)$is analogous to the concept of reducts in rough set theory [43]. Moreover, the intersection of all the sets $\mathrm{Syn}, \mathrm{Syn}^{+}$, and of all the sets $\mathrm{Syn}^{-}$, is analogous to the concept of core in rough set theory [43].

\section{Further extensions}

The three-phase method described above can be considered a standard procedure; first, we check the existence of a utility function of type (3) compatible with the customers' answers (phase (i)), then we look for a minimal set of couples of interacting criteria (phase (ii)), and finally we look for a utility function having the maximum discrimination power (phase (iii)). In this section, some interesting extensions of this procedure are presented.

\subsection{Finding other minimal sets of couples of interacting criteria}

In general, there may exist more than one minimal set Syn or minimal pair $\left(S y n^{+}, S y n^{-}\right)$and, for this reason, it could be interesting to find them all.

In case of option (S1), in order to find another minimal set $S y n_{2}$, one has to solve the following optimization problem:

$$
\begin{gathered}
\text { Minimize: } \sum_{\{i, j\} \in I^{(2)}} \gamma_{i j} \text {, s.t. } \\
\left.\sum_{\{i, j\} \in \text { Syn }_{1}} \gamma_{i j} \leq\left|S y n_{1}\right|-1\right\}\left(E_{M_{2}}\right)
\end{gathered}
$$

where $S y n_{1}$ is the set of interacting criteria found in phase (ii). The last constraint in $\left(E_{M_{2}}\right)$ ensures that a newly set of indices of couples of interacting criteria is different from the previous one.

Let us suppose that at the $(k-1)^{t h}$ iteration we found the minimal set $S y n_{k-1}$. In order to check if there exists another minimal set $S y n_{k}$, it will be sufficient to solve the following optimization problem:

$$
\left.\begin{array}{c}
\text { Minimize: } \sum_{\{i, j\} \in I^{(2)}} \gamma_{i j} \text {, s.t. } \\
\left(E_{2}\right), \\
\sum_{\{i, j\} \in S y n_{1}} \gamma_{i j} \leq\left|S y n_{1}\right|-1, \\
\sum_{\{i, j\} \in S_{y n}} \gamma_{i j} \leq\left|S y n_{2}\right|-1, \\
\sum_{\{i, j\} \in S_{y n} n_{k-1}} \gamma_{i j} \leq\left|S y n_{k-1}\right|-1
\end{array}\right\}\left(E_{M_{k}}\right)
$$

If problem (15) is infeasible, then there is no minimal set $S y n_{k}$, so that the set $M$ of all minimal sets Syn is given by 


$$
M=\left\{\operatorname{Syn}_{1}, \cdots, \text { Syn }_{k-1}\right\} .
$$

If, instead, problem (15) is feasible, then $S y n_{k}$ is a new minimal pair with

$$
\operatorname{Syn}_{k}=\left\{\{i, j\} \in I^{(2)}: \gamma_{i j}=1 \text { in the solution of problem }(15)\right\}
$$

In case of options $(\mathbf{S 2})$ and $(\mathbf{S 3})$, in order to find another minimal pair $\left(S y n_{2}^{+}, S y n_{2}^{-}\right)$, one has to solve the following optimization problem:

$$
\begin{gathered}
\text { Minimize: } \sum_{\{i, j\} \in I^{(2)}}\left(\delta_{i j}^{+}+\delta_{i j}^{-}\right), \text {s.t. } \\
\left.\sum_{\{i, j\} \in\left\{S y n_{1}^{+} \cup S y n_{1}^{-}\right\}}^{\left(E_{2}\right),}\left(\delta_{i j}^{+}+\delta_{i j}^{-}\right) \leq\left|S y n_{1}^{+} \cup S y n_{1}^{-}\right|-1\right\}\left(E_{M_{2}}\right)
\end{gathered}
$$

where $S y n_{1}^{+}$and $S y n_{1}^{-}$are the sets of couples of positively and negatively interacting criteria found in phase (ii).

Let us suppose that at the $(k-1)^{t h}$ iteration we found the minimal pair $\left(S y n_{k-1}^{+}, S y n_{k-1}^{-}\right)$. In order to check if there exists another minimal pair $\left(S y n_{k}^{+}, S y n_{k}^{-}\right)$, it will be sufficient to solve the following optimization problem:

$$
\left.\begin{array}{c}
\text { Minimize: } \sum_{\{i, j\} \in I^{(2)}}\left(\delta_{i j}^{+}+\delta_{i j}^{-}\right) \text {, s.t. } \\
\sum_{\{i, j\} \in\left\{\text { Syn }_{1}^{+} \cup S y n_{1}^{-}\right\}}^{\left(E_{2}\right),}\left(\delta_{i j}^{+}+\delta_{i j}^{-}\right) \leq\left|S y n_{1}^{+} \cup S y n_{1}^{-}\right|-1, \\
\sum_{\{i, j\} \in\left\{S y n_{2}^{+} \cup S y n_{2}^{-}\right\}}\left(\delta_{i j}^{+}+\delta_{i j}^{-}\right) \leq\left|S y n_{2}^{+} \cup S y n_{2}^{-}\right|-1, \\
\sum_{\{i, j\} \in\left\{S y n_{k-1}^{+} \cup S y n_{k-1}^{-}\right\}}\left(\delta_{i j}^{+}+\delta_{i j}^{-}\right) \leq\left|S y n_{k-1}^{+} \cup S y n_{k-1}^{-}\right|-1
\end{array}\right\}\left(E_{\left.M_{k}\right)}\right.
$$

If problem (17) is infeasible, then there is no minimal pair $\left(S y n_{k}^{+}, S y n_{k}^{-}\right)$, so that the set $M$ of all minimal pairs $\left(S y n^{+}, S y n^{-}\right)$is given by

$$
M=\left\{\left(\operatorname{Syn}_{1}^{+}, \operatorname{Syn}_{1}^{-}\right), \cdots,\left(\operatorname{Syn}_{k-1}^{+}, \operatorname{Syn}_{k-1}^{-}\right)\right\} .
$$

If, instead, problem (17) is feasible, then $\left(S y n_{k}^{+}, S y n_{k}^{-}\right)$is a new minimal pair with

$$
\operatorname{Syn}_{k}^{+}=\left\{\{i, j\} \in I^{(2)}: \delta_{i j}^{+}=1 \text { in the solution of problem }(17)\right\}
$$

and

$$
\operatorname{Syn}_{k}^{-}=\left\{\{i, j\} \in I^{(2)}: \delta_{i j}^{-}=1 \text { in the solution of problem (17) }\right\} .
$$




\subsection{Customer satisfaction evaluation using a set of compatible prefer- ence models}

When analyzing the customers' survey presented in Table 1, the experts of the company could be interested to know what action should be made in order to improve the customer satisfaction of the service or product provided. For example, let us consider a service evaluated on three criteria, purchase process, product and additional service, expressed on three different levels of satisfaction: Dissatisfied, Satisfied and Very Satisfied. The experts could be interested in answering the following question: "is the profile of customer satisfaction $P_{1}=$ (Satisfied, Very Satisfied, Satisfied) appreciated more than the profile of customer satisfaction $P_{2}=$ (Very Satisfied, Satisfied, Very Satisfied)?" To answer this question, the experts have to consider the set $\mathcal{U}$ of utility functions of type (3) satisfying the set of constraints $\left(E_{1}\right)$ related to the considered customers' survey. Such utility functions are called compatible with customers' preferences. Then, a natural question arises whether profile $P_{1}$ is at least as good as profile $P_{2}$ for at least one or for all compatible utility functions from $\mathcal{U}$. By answering this type of questions, the experts can get an additional insight into more meaningful decision investments concerning the service or product provided by the company. For example, if the experts find that profile $P_{1}$ is better than profile $P_{2}$ for all compatible utility functions from $\mathcal{U}$, they can conclude that an action directed to increase satisfaction level from Satisfied to Very Satisfied on 'purchase process' is likewisely more appreciated by the customers than another action directed to an analogous improvement on both 'product' and 'additional service'. To perform this type of analysis, one can use the Robust Ordinal Regression (ROR) methodology being a family of MCDA methods introduced in [22] (for a recent survey on the topic see [25]). ROR has been applied to ranking problems (see UTA ${ }^{G M S}[22]$, GRIP [11]), to sorting problems (see UTADIS ${ }^{G M S}$ [23]), and also in methods using outranking relations (ELECTRE ${ }^{G K M S}[17]$ ) or Choquet integral (NAROR [3]) as preference models.

Given an initial set of preference information provided by a Decision Maker (DM), the ROR aims at obtaining a final recommendation for the decision problem at hand, taking into account not only one preference model compatible with this preference information, but the whole set of compatible preference models simultaneously. In fact, as it is often the case in the inference procedures, several decision models could be compatible with the information provided by the DM, but each one of them could lead to different preferences on the remaining alternatives, not considered by the DM at the stage of expressing the preference information. The choice of one particular preference model among all compatible ones could be considered arbitrary, and so it is more meaningful to take into account the whole set of compatible preference models simultaneously. Supposing the preference model in the form of a set of compatible utility functions, the conclusions drawn by ROR are based on two preference relations:

- the necessary preference relation for which alternative $a$ is necessarily preferred to alternative $b$, if $a$ is at least as good as $b$ for all utility functions compatible with the preference information provided by the DM,

- the possible preference relation for which alternative $a$ is possibly preferred to alternative $b$ if $a$ is at least as good as $b$ for at least one utility function compatible with the preference information provided by the DM.

In the following, we adapt the concept of ROR to MUSA-INT considering the following binary relations on the set of profiles $\mathcal{L}=\prod_{i=1}^{n} \mathcal{L}_{i}$. Given any two profiles, $P_{1}, P_{2} \in \mathcal{L}$ :

- profile of customer satisfaction $P_{1}$ is possibly preferred to profile $P_{2},\left(P_{1} \succsim^{P} P_{2}\right)$, if profile $P_{1}$ is at least as good as profile $P_{2}$ for at least one compatible utility function of $\mathcal{U}$, 
- profile of customer satisfaction $P_{1}$ is necessarily preferred to profile $P_{2},\left(P_{1} \succsim^{N} P_{2}\right)$, if profile $P_{1}$ is at least as good as profile $P_{2}$ for all compatible utility functions of $\mathcal{U}$.

Let us stress that in our context, there is no DM but only an analyst supporting the experts of the company in the analysis of customer satisfaction; for this reason the preference information provided by the DM in the ROR context is replaced by the answers to the customers' survey (expressed by set of constraints $\left.\left(E_{*}\right)\right)$ in our method.

Analogously, the concept of compatible utility function in this context is slightly different from the one used in ROR. Here, we call compatible a utility function obtained for an approximation error lower or equal to maxerror, while in the classical ROR methods, a preference model is called compatible with the preference information provided by the DM when it can represent this information without any error.

In order to compute the necessary and possible preference relations between two profiles of customer satisfaction $\left(s a t_{a, 1}, s a t_{a, 2}, \ldots, s a t_{a, n}\right)$ and $\left(s a t_{b, 1}, s a t_{b, 2}, \ldots, s a t_{b, n}\right)$, one should proceed in the following way.

Considering the sets of constraints,

$$
\left.\begin{array}{l}
\sum_{i=1}^{n} u_{i}\left(\operatorname{sat}_{b, i}\right)+\sum_{\{i, j\} \in I^{(2)}} \operatorname{syn}_{i j}^{+}\left(\operatorname{sat}_{b, i}, \operatorname{sat}_{b, j}\right)-\sum_{\{i, j\} \in I^{(2)}} \operatorname{syn}_{i j}^{-}\left(\operatorname{sat}_{b, i}, \operatorname{sat}_{b, j}\right) \geq \\
\geq \sum_{i=1}^{n} u_{i}\left(\operatorname{sat}_{a, i}\right)+\sum_{\{i, j\} \in I^{(2)}} \operatorname{syn}_{i j}^{+}\left(\operatorname{sat}_{a, i}, s a t_{a, j}\right)-\sum_{\{i, j\} \in I^{(2)}} \operatorname{syn}_{i j}^{-}\left(\operatorname{sat}_{a, i}, s a t_{a, j}\right)+\varepsilon, \\
\left(E_{1}\right), \\
\sum_{c \in C}\left(\sigma_{c}^{+}+\sigma_{c}^{-}\right) \leq \text {maxerror, }
\end{array}\right\} E^{N}(a, b)
$$

and

$$
\left.\begin{array}{l}
\sum_{i=1}^{n} u_{i}\left(\operatorname{sat}_{a, i}\right)+\sum_{\{i, j\} \in I^{(2)}} \operatorname{syn}_{i j}^{+}\left(\operatorname{sat}_{a, i}, s_{a, j}\right)-\sum_{\{i, j\} \in I^{(2)}} \operatorname{syn}_{i j}^{-}\left(\operatorname{sat}_{a, i}, s_{a, j}\right) \geq \\
\geq \sum_{i=1}^{n} u_{i}\left(\operatorname{sat}_{b, i}\right)+\sum_{\{i, j\} \in I^{(2)}} \operatorname{syn}_{i j}^{+}\left(\operatorname{sat}_{b, i}, s a t_{b, j}\right)-\sum_{\{i, j\} \in I^{(2)}} \operatorname{syn}_{i j}^{-}\left(\operatorname{sat}_{b, i}, s a t_{b, j}\right), \\
\left(E_{1}\right), \\
\sum_{c \in C}\left(\sigma_{c}^{+}+\sigma_{c}^{-}\right) \leq \text {maxerror },
\end{array}\right\} E^{P}(a, b)
$$

where maxerror is the maximum accepted total approximation error, and $\varepsilon$ in the constraint $U\left(\ell_{p}^{n+1}\right) \geq U\left(\ell_{p-1}^{n+1}\right)+\varepsilon$ is an auxiliary variable, one can conclude the following:

- profile $\left(s a t_{a, 1}, s a t_{a, 2}, \ldots, s a t_{a, n}\right)$ is necessarily preferred to profile $\left(s_{b, 1}, s a t_{b, 2}, \ldots, s_{b, n}\right)$ if $E^{N}(a, b)$ is infeasible or $\varepsilon^{N} \leq 0$, where $\varepsilon^{N}=\max \varepsilon$, subject to $E^{N}(a, b)$,

- $\operatorname{profile}\left(s a t_{a, 1}, s a t_{a, 2}, \ldots, s a t_{a, n}\right)$ is possibly preferred to profile $\left(s a t_{b, 1}, s_{b} t_{b, 2}, \ldots, s_{t} t_{b, n}\right)$ if $E^{P}(a, b)$ is feasible and $\varepsilon^{P}>0$, where $\varepsilon^{P}=\max \varepsilon$, subject to $E^{P}(a, b)$.

Analogous conclusions can be drawn using a set of approximately compatible utility functions. Then, one has to consider the following two sets of constraints: 


$$
\begin{aligned}
& \sum_{i=1}^{n} u_{i}\left(s a t_{b, i}\right)+\sum_{\{i, j\} \in I^{(2)}} \operatorname{syn}_{i j}^{+}\left(\operatorname{sat}_{b, i}, s a t_{b, j}\right)-\sum_{\{i, j\} \in I^{(2)}} s y n_{i j}^{-}\left(s_{a t} t_{b, i}, s a t_{b, j}\right)+\sigma_{1}^{+}-\sigma_{1}^{-} \geq \\
& \geq \sum_{i=1}^{n} u_{i}\left(s a t_{a, i}\right)+\sum_{\{i, j\} \in I^{(2)}} \operatorname{syn}_{i j}^{+}\left(s a t_{a, i}, s a t_{a, j}\right)-\sum_{\{i, j\} \in I^{(2)}} \operatorname{syn}_{i j}^{-}\left(s a t_{a, i}, s a t_{a, j}\right)+\sigma_{2}^{+}-\sigma_{2}^{-}+\varepsilon, \\
& \left(E_{1}\right) \text {, } \\
& \sum_{c \in C}\left(\sigma_{c}^{+}+\sigma_{c}^{-}\right) \leq \text {maxerror, } \\
& \sigma_{1}^{+}+\sigma_{1}^{-} \leq o p t_{1}, \\
& \sigma_{2}^{+}+\sigma_{2}^{-} \leq o p t_{2}
\end{aligned}
$$

and

$$
\left.\begin{array}{l}
\sum_{i=1}^{n} u_{i}\left(s a t_{a, i}\right)+\sum_{\{i, j\} \in I^{(2)}} \operatorname{syn}_{i j}^{+}\left(\operatorname{sat}_{a, i}, s a t_{a, j}\right)-\sum_{\{i, j\} \in I^{(2)}} \operatorname{syn}_{i j}^{-}\left(\operatorname{sat}_{a, i}, s a t_{a, j}\right)+\sigma_{1}^{+}-\sigma_{1}^{-} \geq \\
\geq \sum_{i=1}^{n} u_{i}\left(s^{2} t_{b, i}\right)+\sum_{\{i, j\} \in I^{(2)}} \operatorname{syn}_{i j}^{+}\left(\operatorname{sat}_{b, i}, s a t_{b, j}\right)-\sum_{\{i, j\} \in I^{(2)}} \operatorname{syn}_{i j}^{-}\left(\operatorname{sat}_{b, i}, s a t_{b, j}\right)+\sigma_{2}^{+}-\sigma_{2}^{+}, \\
\left(E_{1}\right), \\
\sum_{c \in C}\left(\sigma_{c}^{+}+\sigma_{c}^{-}\right) \leq \text {maxerror, } \\
\sigma_{1}^{+}+\sigma_{1}^{-} \leq \text {opt }_{1}, \\
\sigma_{2}^{+}+\sigma_{2}^{-} \leq \text {opt }_{2},
\end{array}\right\} E_{1}^{P}(a, b)
$$

where $\sigma_{1}^{+}, \sigma_{1}^{-}, \sigma_{2}^{+}$, and $\sigma_{2}^{-}$are, error variables of the utility values relative to profiles $a$ and $b$, while $o p t_{1}$ and $o p t_{2}$ represent the maximum accepted errors in each one of the considered profile's utility.

Since the new set of constraints, $E_{1}^{N}(a, b)$ and $E_{1}^{P}(a, b)$, enlarge the decision space of the utility functions compatible with the customers' preferences, the following two preference relations, analogous to the ones introduced above, are defined:

- a strong necessary preference relation, for which $a$ is strongly necessarily preferred to $b$ if $a$ is at least as good as $b$ for all utility functions approximately compatible with the customers' preferences,

- a weak possible preference relation, for which $a$ is weakly possibly preferred to $b$ if $a$ is at least as good as $b$ for at least one utility function approximately compatible with the customers' preferences.

Let us remark that we have considered two different qualifications (strong and weak) for the necessary and possible preference representation. In fact, since taking into account the error variables enlarges the set of compatible utility functions, if $a$ is at least as good as $b$ with respect to all approximately compatible utility functions, then $a$ is strongly necessarily preferred to $b$. On the contrary, if $a$ is not at least as good as $b$ for any compatible model in case error variables are considered, then even considering utility functions admitting some error, we cannot find any utility function for which $a$ is at least as good as $b$. 


\section{Illustrative example}

We shall illustrate MUSA-INT using an example originally considered by Grigoroudis and Siskos [30], concerning 20 customers evaluating a service provided by an enterprise. In order to show the full potential of our method, we have augmented the customer dataset presented in [30] by 4 customers, denoted by $x, y, w$, and $z$. The main features of our illustrative example are listed hereafter:

1) evaluation of the service involves three criteria concerning: product (1), purchase process (2) and additional service (3);

2) three levels of satisfaction (Very Satisfied (V), Satisfied (S), Dissatisfied (D)) are considered with respect to both, every criterion and comprehensive satisfaction of the service;

3) the customer's satisfaction survey is composed of answers provided by 24 customers and displayed in Table 1.

In the following, we will play the role of the analyst supporting the customer satisfaction expert.

Table 1: Consumers' satisfaction survey

\begin{tabular}{|c|c|c|c|c|}
\hline Customer & Comprehensive satisfaction & Product (1) & Purchase process (2) & Additional service (3) \\
\hline \hline 1 & Satisfied & Very Satisfied & Satisfied & Dissatisfied \\
2 & Dissatisfied & Dissatisfied & Dissatisfied & Dissatisfied \\
3 & Very Satisfied & Very Satisfied & Very Satisfied & Very Satisfied \\
4 & Satisfied & Very Satisfied & Dissatisfied & Dissatisfied \\
5 & Dissatisfied & Dissatisfied & Dissatisfied & Very Satisfied \\
6 & Very Satisfied & Very Satisfied & Very Satisfied & Very Satisfied \\
7 & Satisfied & Very Satisfied & Dissatisfied & Very Satisfied \\
8 & Satisfied & Very Satisfied & Dissatisfied & Satisfied \\
9 & Satisfied & Satisfied & Satisfied & Dissatisfied \\
10 & Dissatisfied & Dissatisfied & Dissatisfied & Dissatisfied \\
11 & Satisfied & Satisfied & Very Satisfied & Dissatisfied \\
12 & Dissatisfied & Dissatisfied & Dissatisfied & Very Satisfied \\
13 & Very Satisfied & Very Satisfied & Very Satisfied & Dissatisfied \\
14 & Satisfied & Satisfied & Very Satisfied & Dissatisfied \\
15 & Dissatisfied & Dissatisfied & Dissatisfied & Satisfied \\
16 & Very Satisfied & Very Satisfied & Very Satisfied & Very Satisfied \\
17 & Very Satisfied & Very Satisfied & Very Satisfied & Satisfied \\
18 & Very Satisfied & Very Satisfied & Very Satisfied & Satisfied \\
19 & Satisfied & Satisfied & Satisfied & Dissatisfied \\
20 & Dissatisfied & Satisfied & Dissatisfied & Satisfied \\
$\mathrm{x}$ & Very Satisfied & Satisfied & Very Satisfied & Very Satisfied \\
y & Satisfied & Satisfied & Satisfied & Satisfied \\
w & Dissatisfied & Dissatisfied & Very Satisfied & Very Satisfied \\
z & Satisfied & Dissatisfied & Satisfied \\
\hline
\end{tabular}

For customers $x, y, w$, and $z$, it is easy to show that the axiom of the preferential independence is violated [39].

Supposing that the utility function of all the customers has an additive form and does not handle synergies between criteria, we can observe the following:

1) since customers $x$ and $y$ have the same levels of satisfaction with respect to criterion 'product', and the comprehensive satisfaction level of $x$ is greater than the comprehensive satisfaction level of $y$, we get:

$$
u_{1}(x)+u_{2}(x)+u_{3}(x)>u_{1}(y)+u_{2}(y)+u_{3}(y) \Rightarrow u_{2}(x)+u_{3}(x)>u_{2}(y)+u_{3}(y)
$$

2) since customers $w$ and $z$ have the same levels of satisfaction with respect to criterion 'product', and the comprehensive satisfaction level of $w$ is lower than the comprehensive satisfaction level of $z$, we get:

$$
u_{1}(w)+u_{2}(w)+u_{3}(w)<u_{1}(z)+u_{2}(z)+u_{3}(z) \Rightarrow u_{2}(w)+u_{3}(w)<u_{2}(z)+u_{3}(z)
$$


3) since customers $x$ and $w$ have the same levels of satisfaction with respect to criteria 'purchase process' and 'additional service', and customers $y$ and $z$ have the same levels of satisfaction with respect to criteria 'purchase process' and 'additional service', we obtain:

$$
u_{2}(x)+u_{3}(x)=u_{2}(w)+u_{3}(w) \text { and } u_{2}(y)+u_{3}(y)=u_{2}(z)+u_{3}(z) .
$$

From 1), 2) and 3) we get a contradiction since at the same time it should be true that $u_{2}(x)+u_{3}(x)>$ $u_{2}(y)+u_{3}(y)$ and $u_{2}(x)+u_{3}(x)<u_{2}(y)+u_{3}(y)$.

As a result, we conclude that the customers' comprehensive satisfaction cannot be represented by an additive utility function, and thus, the MUSA method using this type of utility function is not able to fully represent the comprehensive satisfaction of the customers shown in Table 1.

For this reason, to represent the customers' comprehensive satisfaction shown in Table 1, we apply MUSA-INT, adopting a utility function with positive and negative synergy components (3). In the following we shall consider option (S1) only, that is the case where there could exist both positive and negative interactions for the same couple of criteria. The computations for the other two options ((S2) and (S3)) can be done analogously.

As described in phase (i), we would like to consider the simplest possible utility function, that is the one involving the minimum number of interactions. For this reason, to start we suppose that each criterion can interact with at most one another criterion. Solving the MILP problem (9) in which $\varepsilon$ has been fixed to 0.1 , we get the total approximation error opter $=0.5$. This means that, under the hypothesis that each criterion can interact with at most one another criterion, there does not exist any utility function of type (3) representing the satisfaction of all customers.

Since maxerror, indicating the maximum acceptable error, is set equal to 0.3 , we decide to increase the maximum number of criteria with which each criterion can interact. This means that now each criterion can interact with at most two other criteria and not only one, as before. In consequence of this assumption, constraints of type (10) should be added to the set of constraints $E_{*}$. After introducing all these constraints, and solving again MILP problem (9), we get $\sigma_{c}^{+}=\sigma_{c}^{-}=0$ for all $c \in$ $C$, and therefore opterr $=0$. This time, there exist a utility function of type (3) representing the satisfaction of all customers. From the MILP problem (9), we obtain $\gamma_{12}=\gamma_{13}=1$ that is Syn $=\{\{1,2\},\{1,3\}\}$; this means that criterion 'product' (1) interacts with both criteria 'purchase process' (2) and 'additional service' (3).

After fixing $\alpha=0$, that is no admitting any deterioration of the total admissible error, in phase (ii) (see Section 3.2), we solve MILP problem (11) to determine a set of couples of interacting criteria. Solving the MILP problem (11), we get the same interactions as we found previously. This means that the set Syn of couples of criteria is the minimum one with respect to the inclusion.

In phase (iii) (see Section 3.3), we proceed in two steps to find the most discriminating utility function. In the first step, when maximizing the discrimination of satisfaction levels of the comprehensive utility, we find $\varepsilon_{\text {comprehensive }}=0.5$ while the utility function and the interactions are shown in Table 2 . In this case, the couples of criteria $\{1,2\}$ and $\{1,3\}$ present only positive interactions while couple of criteria $\{2,3\}$ is not interacting.

At this point, in the second step, in order to maximize the discrimination of satisfaction levels of the marginal utilities we solve the MILP problem (13) finding the utility function shown in Table 3. Looking at Table $3(\mathrm{~b})$, we observe that couples of criteria $\{1,2\}$ and $\{1,3\}$ present positive and negative interactions while the couple of criteria $\{2,3\}$ is not interacting. In particular, comparing the levels of the interactions for each pair of levels, we observe that the negative interaction is greater or equal to the positive interaction. For example, looking at the interactions of the pair $(V, D)$ for the couple of criteria $\{1,3\}$, we have $\operatorname{syn}_{13}^{+}(V, D)=0.1$ and $\operatorname{syn}_{13}^{-}(V, D)=0.4$.

In phase (ii) of the illustrative example presented above, we found the minimal set $\operatorname{Syn}_{1}=\{(1,2),(1,3)\}$. Looking for other minimal sets, we solve the MILP problem (14) that does not give other solutions. 
Table 2: Parameters of the most discriminating utility function resulting from optimal solution of MILP problem (12)

(a) Marginal utilities and interactions

\begin{tabular}{|c|c|c|c|c|}
\hline & $u_{1}$ & $u_{2}$ & $u_{3}$ & $\mathrm{U}$ \\
\hline $\mathrm{D}$ & 0 & 0 & 0 & 0 \\
$\mathrm{~S}$ & 0 & 0 & 0 & 0.5 \\
$\mathrm{~V}$ & 0 & 0 & 0 & 1 \\
\hline
\end{tabular}

\begin{tabular}{|c|c|c|}
\hline$\gamma_{12}$ & $\gamma_{13}$ & $\gamma_{23}$ \\
\hline 1 & 1 & 0 \\
\hline
\end{tabular}

(b) Values of the interactions for all pairs of levels

\begin{tabular}{|c|c|c|c|c|c|c|}
\hline & $\mathrm{syn}_{12}^{+}$ & $\mathrm{syn}_{12}^{-}$ & $\mathrm{syn}_{13}^{+}$ & $\mathrm{syn}_{13}^{-}$ & $\mathrm{syn}_{23}^{+}$ & $\mathrm{syn}_{23}^{-}$ \\
\hline \hline VV & 0.5 & 0 & 0.5 & 0 & 0 & 0 \\
VS & 0 & 0 & 0.5 & 0 & 0 & 0 \\
VD & 0 & 0 & 0.5 & 0 & 0 & 0 \\
$\mathrm{SV}$ & 0.5 & 0 & 0.5 & 0 & 0 & 0 \\
$\mathrm{SS}$ & 0 & 0 & 0.5 & 0 & 0 & 0 \\
$\mathrm{SD}$ & 0 & 0 & 0 & 0 & 0 & 0 \\
$\mathrm{DV}$ & 0 & 0 & 0.5 & 0 & 0 & 0 \\
$\mathrm{DS}$ & 0 & 0 & 0 & 0 & 0 & 0 \\
\hline $\mathrm{DD}$ & 0 & 0 & 0 & 0 & 0 & 0 \\
\hline
\end{tabular}

Table 3: Parameters of the most discriminating utility function resulting from optimal solution of MILP problem (13)

(a) Marginal utilities and interactions

\begin{tabular}{|c|c|c|c|c|}
\hline & $u_{1}$ & $u_{2}$ & $u_{3}$ & $\mathrm{U}$ \\
\hline $\mathrm{D}$ & 0 & 0 & 0 & 0 \\
$\mathrm{~S}$ & 0.6 & 0.4 & 0.4 & 0.5 \\
$\mathrm{~V}$ & 1 & 0.8 & 0.8 & 1 \\
\hline
\end{tabular}

\begin{tabular}{|c|c|c|}
\hline$\gamma_{12}$ & $\gamma_{13}$ & $\gamma_{23}$ \\
\hline 1 & 1 & 0 \\
\hline
\end{tabular}

(b) Values of the interactions for all pairs of levels

\begin{tabular}{|c|c|c|c|c|c|c|}
\hline & $\mathrm{syn}_{12}^{+}$ & $\mathrm{Syn}_{12}^{-}$ & $\mathrm{syn}_{13}^{+}$ & $\mathrm{syn}_{13}^{-}$ & $\mathrm{syn}_{23}^{+}$ & $\mathrm{Syn}_{23}^{-}$ \\
\hline \hline VV & 0.3 & 1 & 0.1 & 1 & 0 & 0 \\
VS & 0 & 0.6 & 0.1 & 0.6 & 0 & 0 \\
VD & 0 & 0.4 & 0.1 & 0.4 & 0 & 0 \\
SV & 0.3 & 0.8 & 0.1 & 0.8 & 0 & 0 \\
SS & 0 & 0.6 & 0.1 & 0.4 & 0 & 0 \\
SD & 0 & 0.2 & 0 & 0.4 & 0 & 0 \\
DV & 0 & 0.8 & 0.1 & 0.4 & 0 & 0 \\
DS & 0 & 0.4 & 0 & 0.4 & 0 & 0 \\
\hline DD & 0 & 0 & 0 & 0 & 0 & 0 \\
\hline
\end{tabular}

This means that $S y n_{1}$ is the only minimal set of couples of interacting criteria and therefore the satisfaction of the customers can be described in a unique way, that is considering positive and negative interaction for the couples of criteria $\{1,2\}$ and $\{1,3\}$. Applying the ROR as described in Section 4.2 , and after fixing $o p t_{1}=o p t_{2}=0.1$, we show here some interesting observations:

- profile $(D, S, V)$ is strongly necessarily preferred to profile $(D, V, S)$,

- profile $(S, V, D)$ is necessarily preferred but not strongly necessarily preferred to profile $(V, D, S)$,

- profile $(V, S, D)$ is neither weakly possibly nor possibly preferred to profile $(S, V, S)$.

The first result, for example, could be interpreted by saying that considering "Dissatisfied" level for 'product', the customers prefer to have "Satisfied" level for 'purchase process' and "Very Satisfied" level for 'additional service' rather than "Very Satisfied" level for 'purchase product' and "Satisfied" level for 'additional service'. 


\section{Conclusions}

In this paper, we proposed MUSA-INT, a new multicriteria customer satisfaction analysis method able to take into account positive and negative interactions among criteria, even if the customers' judgments are qualitative and not quantitative. To explain the customer's preferences, the method employs an additive utility function augmented with components representing positive and negative interactions between two satisfaction levels of two criteria.

Some strong points of our method are listed hereafter:

- the criteria are expressed on ordinal scales, without the necessity of expressing all the criteria on a common ordinal or cardinal scale, as this is the case of the Choquet integral or some other fuzzy integrals;

- the model reveals the interactions among criteria in the customer satisfaction evaluation of a product or a service;

- the interactions among criteria have a meaningful interpretation for the customer as a bonus (for positive interaction) or penalty (for negative interaction), added to or subtracted from the sum of marginal utility values;

- there is a parsimonious representation of the interactions by considering minimal pairs of sets of couples of interacting criteria;

- one can identify all minimal pairs of sets of couples of interacting criteria;

- as the preference model (utility function) representing the customers' satisfaction is, in general, not unique, it is possible to take into account the whole set of compatible preference models adopting the Robust Ordinal Regression methodology.

We envisage some possible directions of future research:

(1) Consideration of positive or negative interaction not only between couples of criteria, but also triples, quadruples and, generally, sets of criteria of cardinality greater than 2 . Using the example of a supermarket, it may be reasonable to admit that there is a specific surplus in the appreciation due to the presence at the same time of low prices, special offers and quality. In this case, the considered utility function will become

$$
U\left(s a t_{c, n+1}\right)=\sum_{i=1}^{n} u_{i}\left(\operatorname{sat}_{c, i}\right)+\sum_{A \in S y n_{G}^{+}} \operatorname{syn}_{A}^{+}\left(\operatorname{sat}_{c, i}, i \in A\right)-\sum_{A \in S y n_{G}^{-}} \operatorname{syn}_{A}^{-}\left(\operatorname{sat}_{c, i}, i \in A\right), c \in C
$$

where $\operatorname{Syn}_{G}^{+}, \operatorname{Syn}_{G}^{-} \subseteq 2^{I}$ are the families of all the subsets of criteria for which there is a positive interaction and a negative interaction, respectively. Considerations of interaction among criteria in subsets with cardinality greater than 2 requires to pay a specific attention to the tradeoff between the better knowledge one gets about customer satisfaction and the additional computational effort required to get this knowledge.

(2) The representation of customers' preferences using an outranking model instead of a utility function; in this case, the interaction can be represented taking into account the concordance index of ELECTRE method presented in [10], or the bipolar PROMETHEE proposed in [6]. 
(3) Consideration of a hierarchal structure of criteria in the customers' survey. Indeed, very often the customers are required to evaluate features of a product or a service organized in a hierarchical way. For instance, taking into account our illustrative example, product satisfaction could be split into satisfaction with respect to aspects $A_{1}, A_{2}$ and $A_{3}$, so that we have an evaluation on the three aspects and a comprehensive evaluation with respect to 'product'. A similar level of detail can be considered for 'purchase product' and 'additional service'. In this case, we could consider an ordinal regression approach concordant with the principle of Multiple Criteria Hierarchy Process $[2,7,8]$.

(4) Application of all ROR extensions, such as SMAA applied to Robust Ordinal Regression [38].

\section{Acknowledgments}

The fourth author wishes to acknowledge financial support from the Polish National Science Center, grant no. 155534 .

\section{References}

[1] L.S. Aiken and S.G. West. Multiple regression: Testing and interpreting interactions. Sage, Newbury Park, CA, 1991.

[2] S. Angilella, S. Corrente, S. Greco, and R. Słowiński. Multiple Criteria Hierarchy Process for the Choquet integral. In R.C. Purshouse et al., editor, EMO 2013, volume 7811 of LNCS, pages 475-489. Springer Berlin Heidelberg, 2013.

[3] S. Angilella, S. Greco, and B. Matarazzo. Non-additive robust ordinal regression: A multiple criteria decision model based on the Choquet integral. European Journal of Operational Research, 201(1):277-288, 2010.

[4] J.D. Carroll and P.E. Green. Guest editorial: Psychometric methods in marketing research: Part I, Conjoint Analysis. Journal of Marketing Research, 32:385-391, 1995.

[5] G. Choquet. Theory of capacities. Annales de l'Institut Fourier, 5(54):131-295, 1953.

[6] S. Corrente, J.R. Figueira, and S. Greco. Interaction of Criteria and Robust Ordinal Regression in Bi-polar PROMETHEE methods. In S. Greco et al., editor, IPMU 2012, Part IV, CCIS 300, pages 469-479. Springer, Berlin, 2012.

[7] S. Corrente, S. Greco, and R. Słowiński. Multiple Criteria Hierarchy Process in Robust Ordinal Regression. Decision Support Systems, 53(3):660-674, 2012.

[8] S. Corrente, S. Greco, and R. Słowiński. Multiple Criteria Hierarchy Process with ELECTRE and PROMETHEE. Omega, 41:820-846, 2012.

[9] J.R. Figueira, S. Greco, and M. Ehrgott, editors. Multiple Criteria Decision Analysis: State of the Art Surveys. Springer, Berlin, 2005.

[10] J.R. Figueira, S. Greco, and B. Roy. ELECTRE methods with interaction between criteria: An extension of the concordance index. European Journal of Operational Research, 199(2):478 495, 2009. 
[11] J.R. Figueira, S. Greco, and R. Słowiński. Building a set of additive value functions representing a reference preorder and intensities of preference: GRIP method. European Journal of Operational Research, 195(2):460-486, 2009.

[12] J. Fürnkranz and E. Hüllermeier, editors. Preference Learning. Springer, Berlin, 2010.

[13] A. Gofman. Emergent scenarios, synergies, and suppressions uncovered within conjoint analysis. Journal of Sensory Studies, 21(4):373-414, 2006.

[14] M. Grabisch and C. Labreuche. Bi-capacities-I: definition, Möbius transform and interaction. Fuzzy Sets and Systems, 151(2):211-236, 2005.

[15] M. Grabisch and C. Labreuche. Bi-capacities-II: the Choquet integral. Fuzzy Sets and Systems, 151(2):237-259, 2005.

[16] M. Grabisch and C. Labreuche. Fuzzy measures and integrals in MCDA. In J. Figueira, S. Greco, and M. Ehrgott, editors, Multiple Criteria Decision Analysis: State of the Art Surveys, pages 563-604. Springer, Berlin, 2005.

[17] S. Greco, M. Kadziński, V. Mousseau, and R. Słowiński. ELECTRE ${ }^{G K M S}$ : Robust ordinal regression for outranking methods. European Journal of Operational Research, 214(1):118-135, 2011.

[18] S. Greco, B. Matarazzo, and S. Giove. The Choquet integral with respect to a level dependent capacity. Fuzzy Sets and Systems, 175:1-35, 2011.

[19] S. Greco, B. Matarazzo, and R. Słowiński. Rough sets theory for multicriteria decision analysis. European Journal of Operational Research, 129(1):1-47, 2001.

[20] S. Greco, B. Matarazzo, and R. Słowiński. Customer satisfaction analysis based on rough set approach. Zeitschrift für Betriebswirtschaft, 77:325-339, 2007.

[21] S. Greco, B. Matarazzo, and R. Słowiński. Bipolar Sugeno and Choquet integrals. In EUROFUSE Workshop on Informations Systems, Varenna, Italy, pages 191-196, September 2002.

[22] S. Greco, V. Mousseau, and R. Słowiński. Ordinal regression revisited: multiple criteria ranking using a set of additive value functions. European Journal of Operational Research, 191(2):416436, 2008.

[23] S. Greco, V. Mousseau, and R. Słowiński. Multiple criteria sorting with a set of additive value functions. European Journal of Operational Research, 207(3):1455-1470, 2010.

[24] S. Greco, V. Mousseau, and R. Słowiński. UTA ${ }^{G M S}-I N T$ : robust ordinal regression of value functions handling interacting criteria. European Journal of Operational Research, 239(3):711$730,2014$.

[25] S. Greco, R. Słowiński, V. Mousseau, and J. Figueira. Robust Ordinal Regression. In M. Ehrgott, J. Figueira, and S. Greco, editors, Trends in Multiple Criteria Decision Analysis, pages 241-283. Springer, New York, 2010.

[26] P.E. Green and V. Srinivasan. Conjoint analysis in consumer research: Issues and outlook. Journal of Consumer Research, 5:103-152, 1978.

[27] P.E. Green and Y. Wind. Multiattribute Decision in Marketing: A Measurement Approach. Dryden Press, Hindsale, IL, 1973. 
[28] P.E. Green and Y. Wind. New way to measure consumers' judgements. Harvard Business Review, 53:107-117, 1975.

[29] E. Grigoroudis, Y. Politis, and Y. Siskos. Satisfaction benchmarking and customer classification: An application to the branches of a banking organization. International Transactions in Operational Research, 9(5):599-618, 2002.

[30] E. Grigoroudis and Y. Siskos. Preference disaggregation for measuring and analysing customer satisfaction: The MUSA method. European Journal of Operational Research, 143(1):148-170, 2002 .

[31] E. Grigoroudis and Y. Siskos. A survey of customer satisfaction barometers: Some results from the transportation-communications sector. European Journal of Operational Research, $152(2): 334-353,2004$.

[32] E. Grigoroudis and Y. Siskos. Customer Satisfaction Evaluation. Springer, New York, 2010.

[33] A. Gustafsson, A. Herrmann, and F. Huber. Conjoint analysis as an instrument of market research practice. In A. Gustafsson, A. Herrmann, and F. Huber, editors, Conjoint Measurement. Methods and Applications, pages 5-45. Springer, Berlin, 2000.

[34] L.A. Hayduk and Y.H. Wonnacott. "Effect Equations" or "Effect Coefficients": A note on the visual and verbal presentation of multiple regression interactions. The Canadian Journal of Sociology, 5(4):399-404, 1980.

[35] D.H. Henard and D.M. Szymanski. Customer satisfaction: A meta-analysis of the empirical evidence. Journal of the Academy of Marketing Science, 29(1):16-35, 2001.

[36] Y.C. Hu and C. Hsiao-Chi. Choquet integral-based hierarchical networks for evaluating customer service perceptions on fast food stores. Expert Systems with Applications, 37(12):7880-7887, 2010 .

[37] E. Jacquet-Lagreze and J. Siskos. Assessing a set of additive utility functions for multicriteria decision-making, the UTA method. European Journal of Operational Research, 10(2):151-164, 1982.

[38] M. Kadziński and T. Tervonen. Stochastic ordinal regression for multiple criteria sorting problems. Decision Support Systems, 55(11):55-66, 2013.

[39] R.L. Keeney and H. Raiffa. Decisions with multiple objectives: Preferences and value tradeoffs. J. Wiley, New York, 1976.

[40] J. Lusk and F.B. Norwood. Effect of experimental design on choice-based conjoint valuation estimates. American Journal of Agricultural Economics, 87(3):771-785, 2005.

[41] K. Ogawa. Route choice behavior model using fuzzy integral utility function. Journal of Japan Fuzzy Association, 11(4):690-694, 1999.

[42] R.C. Overton. Moderated multiple regression for interactions involving categorical variables: A statistical control for heterogeneous variance across two groups. Psychological Methods, 6:218$233,2001$.

[43] Z. Pawlak. Rough Sets - Theoretical Aspects of Reasoning about Data. Kluwer Academic, Dordrecht, 1991. 
[44] J.R.A. Ping. A parsimonious estimating technique for interaction and quadratic latent variables. Journal of Marketing Research, 32:336-347, 1995.

[45] F.S. Roberts. Measurement Theory with Applications to Decision-Making, Utility and the Social Sciences. Addison-Wesley, London, 1979.

[46] Y. Siskos, N.F. Matsatsinis, and G. Baourakis. Multicriteria analysis in agricultural marketing: The case of French olive oil market. European Journal of Operational Research, 130(2):315-331, 2001.

[47] A. Torres and J.A. Tribó. Customer satisfaction and brand equity. Journal of Business Research, 64(10):1089 - 1096, 2011.

[48] F.M. Tseng and Y.J. Chiu. Hierarchical fuzzy integral stated preference method for Taiwan's broadband service market. Omega, 33(1):55-64, 2005.

[49] F.M. Tseng and Y.C. Hu. Partitioned fuzzy integral nested logit model for the Taiwan's internet telephony market. International Journal of Computer Mathematics, 86(8):1283-1299, 2009.

[50] F.M. Tseng and C.Y. Yu. Partitioned fuzzy integral multinomial logit model for Taiwan's internet telephony market. Omega, 33(3):267-276, 2005.

[51] P.P. Wakker. Additive representations of preferences: A new foundation of decision analysis, volume 4. Springer, Berlin, 1989.

[52] C.W. Yang. Integrating fuzzy integral with multinomial logit model to evaluate the effects of service quality on traveling airline choice. Information Technology Journal, 9(7):1449-1457, 2010 .

\section{Appendix}

\section{Computational details}

In the following, we shall characterize only the computational effort of the MILP problem (9) solved in phase (i), because the other MILP problems solved in phases (ii) and (iii) have a similar number of constraints and variables.

Considering $r$ customers, $n$ criteria, $s_{i}$ levels for criterion $i$ and $s_{n+1}$ levels of the comprehensive utility we get:

- Variables:

$$
\begin{aligned}
& \text { (S1) } \sum_{i=1}^{n} s_{i}+s_{n+1}+2 \sum_{i=1}^{n-1} \sum_{j=i+1}^{n}\left(s_{i} \cdot s_{j}\right)+2 \cdot r+\left(\begin{array}{l}
n \\
2
\end{array}\right) \\
& \text { (S2) } \sum_{i=1}^{n} s_{i}+s_{n+1}+2 \sum_{i=1}^{n-1} \sum_{j=i+1}^{n}\left(s_{i} \cdot s_{j}\right)+2 \cdot r+2\left(\begin{array}{l}
n \\
2
\end{array}\right), \\
& \text { (S3) } \sum_{i=1}^{n} s_{i}+s_{n+1}+\sum_{i=1}^{n-1} \sum_{j=i+1}^{n}\left(s_{i} \cdot s_{j}\right)+2 \cdot r+\left(\begin{array}{l}
n \\
2
\end{array}\right) .
\end{aligned}
$$

For example, in our case, where $n=3, s_{i}=3, i=1, \ldots, 4$, and there are 24 customers, we have 117 variables for option (S1), 120 variables for option (S2) and 90 variables for option (S3). 
- Constraints:

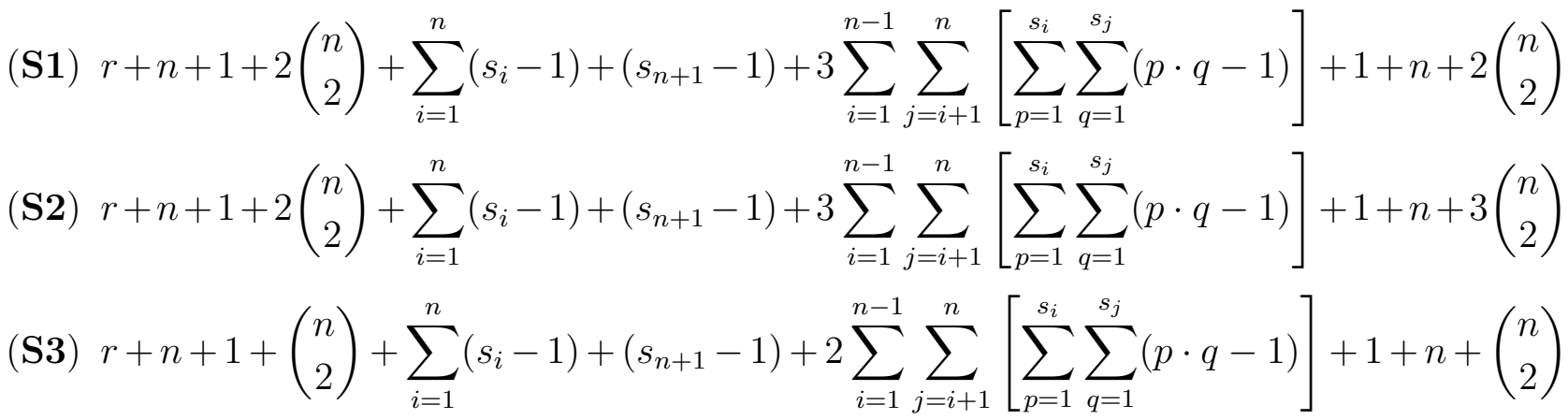

In our example, we have 295 constraints for option (S1), 298 constraints for option (S2) and 208 constraints for option (S3).

Let us observe that the number of constraints can be reduced, as stated also previously in the paper, using the transitivity of $\geq$. For example, let us consider 9 elements in the set $A=\{a, b, c, d, e, f, g, h, i\}$ and a transitive relation $\mathcal{R}$ on the cartesian product $A \times A$, shown in Table 4(a). Let us observe that in Table 4(a), 27 couples belong to $\mathcal{R}$, while in Table 4(b), using the transitivity of $\mathcal{R}$ we have only 12 couples, reducing in this way 15 couples.

Reasoning in this way, we can considerably reduce the number of constraints. In fact, for option (S1) we obtain 160 constraints, i.e. 135 less, while for options (S2) and (S3) we get 163 and 118 constraints, i.e. 135 less and 90 less, respectively.

Table 4: Reduction of the constraints obtained using the transitivity of the relation $\mathcal{R}$

(a) Binary relation on the set $A$

\begin{tabular}{|c|c|c|c|c|c|c|c|c|c|}
\hline $\mathcal{R}$ & $\mathbf{a}$ & $\mathbf{b}$ & $\mathbf{c}$ & $\mathbf{d}$ & $\mathbf{e}$ & $\mathbf{f}$ & $\mathbf{g}$ & $\mathbf{h}$ & $\mathbf{i}$ \\
\hline \hline $\mathbf{a}$ & 0 & 0 & 0 & 0 & 0 & 0 & 0 & 0 & 0 \\
$\mathbf{b}$ & 1 & 0 & 0 & 0 & 0 & 0 & 0 & 0 & 0 \\
$\mathbf{c}$ & 1 & 1 & 0 & 0 & 0 & 0 & 0 & 0 & 0 \\
$\mathbf{d}$ & 1 & 0 & 0 & 0 & 0 & 0 & 0 & 0 & 0 \\
$\mathbf{e}$ & 1 & 1 & 0 & 1 & 0 & 0 & 0 & 0 & 0 \\
$\mathbf{f}$ & 1 & 1 & 1 & 1 & 1 & 0 & 0 & 0 & 0 \\
$\mathbf{g}$ & 1 & 0 & 0 & 1 & 0 & 0 & 0 & 0 & 0 \\
$\mathbf{h}$ & 1 & 1 & 0 & 1 & 1 & 0 & 1 & 0 & 0 \\
$\mathbf{i}$ & 1 & 1 & 1 & 1 & 1 & 1 & 1 & 1 & 1 \\
\hline
\end{tabular}

(b) Minimal couples in the relation $\mathcal{R}$ after using the transitivity

\begin{tabular}{|c|c|c|c|c|c|c|c|c|c|}
\hline & $\mathbf{a}$ & $\mathbf{b}$ & $\mathbf{c}$ & $\mathbf{d}$ & $\mathbf{e}$ & $\mathbf{f}$ & $\mathbf{g}$ & $\mathbf{h}$ & $\mathbf{i}$ \\
\hline \hline $\mathbf{a}$ & 0 & 0 & 0 & 0 & 0 & 0 & 0 & 0 & 0 \\
$\mathbf{b}$ & 1 & 0 & 0 & 0 & 0 & 0 & 0 & 0 & 0 \\
$\mathbf{c}$ & 0 & 1 & 0 & 0 & 0 & 0 & 0 & 0 & 0 \\
$\mathbf{d}$ & 1 & 0 & 0 & 0 & 0 & 0 & 0 & 0 & 0 \\
$\mathbf{e}$ & 0 & 1 & 0 & 1 & 0 & 0 & 0 & 0 & 0 \\
$\mathbf{f}$ & 0 & 0 & 1 & 0 & 1 & 0 & 0 & 0 & 0 \\
$\mathbf{g}$ & 0 & 0 & 0 & 1 & 0 & 0 & 0 & 0 & 0 \\
$\mathbf{h}$ & 0 & 0 & 0 & 0 & 1 & 0 & 1 & 0 & 0 \\
$\mathbf{i}$ & 0 & 0 & 0 & 0 & 0 & 1 & 0 & 1 & 0 \\
\hline
\end{tabular}

